\title{
A comparative study of GPS ionospheric scintillations and ionogram spread F over Sanya
}

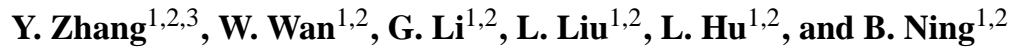 \\ ${ }^{1}$ Key Laboratory of Earth and Planetary Physics, Institute of Geology and Geophysics, \\ Chinese Academy of Sciences, Beijing, China \\ ${ }^{2}$ Beijing National Observatory of Space Environment, Institute of Geology and Geophysics, \\ Chinese Academy of Sciences, Beijing, China \\ ${ }^{3}$ University of Chinese Academy of Sciences, Beijing, China \\ Correspondence to: W. Wan (wanw@mail.iggcas.ac.cn)
}

Received: 14 April 2015 - Revised: 26 October 2015 - Accepted: 3 November 2015 - Published: 19 November 2015

\begin{abstract}
We analyze the data recorded during December 2011-November 2012 by a digital ionosonde and a GPS (Global Positioning System) scintillation and (total electron content) TEC receiver collocated at Sanya $\left(109.6^{\circ} \mathrm{E}\right.$, $18.3^{\circ} \mathrm{N}$; dip lat. $12.8^{\circ} \mathrm{N}$ ), a low-latitude station in the Chinese longitude sector, to carry out a comparative study of ionospheric scintillations and spread F. A good consistency between the temporal variations of GPS scintillation (represented by the S4 index) and of ionogram spread F (represented by the QF index) is found in the pre-midnight period during equinox. However in the post-midnight period during equinox and in the period from post-sunset to presunrise during June solstice, moderate spread F is seen without concurrent GPS scintillation. The possible cause responsible for the difference between post-midnight GPS scintillation and spread $\mathrm{F}$ during equinox could be due to the decaying of $400 \mathrm{~m}$ scale irregularities associated with equatorial spread F. Regarding the irregularities producing moderate QF and low S4 indices during June solstice, we suggest that the frequently observed sporadic E (Es) layer and the medium-scale traveling ionospheric disturbances (MSTIDs) over Sanya could play important roles in triggering the June solstitial spread-F events.
\end{abstract}

Keywords. Ionosphere (ionospheric irregularities)

\section{Introduction}

The ionospheric $\mathrm{F}$ region irregularities are known as electron density inhomogeneous structures, and are mainly generated at night through ionospheric plasma instabilities. The irregularities exist at altitudes from 200 to $1000 \mathrm{~km}$ or even higher, with scale sizes ranging from tens of centimeters to hundreds of kilometers. Numerous means of observations such as satellite in situ and ground-based receivers, radar, rocket, optical imager and ionosondes have been applied to study the spatial and temporal distributions and characteristics of the irregularities during the last few decades at equatorial, low, middle and high latitudes (Abdu et al., 1981; Hysell and Burcham, 1998; Sahai et al., 2000; Su et al., 2008; Lynn et al., 2011).

Equatorial $\mathrm{F}$ region irregularities usually appear as depletions in plasma density evidenced from satellite in situ measurements and are extensively observed at equatorial and low-latitude stations in December in American longitudes, in June solstice in central Pacific longitudes, and at equinox in other longitudes (e.g., Burke et al., 2004; Li et al., 2008; Su et al., 2008). The mechanisms for the generation of equatorial post-sunset irregularities are well established. The RayleighTaylor instability is believed to be the most likely mechanism. The main factors affecting the growth rate of RayleighTaylor instability include the pre-reversal enhancement of eastward electric field (PRE) generated by the coupling of sunset $\mathrm{E}$ and $\mathrm{F}$ layers, the gravity waves and the meridional wind (Abdu, 2001). Earlier ground-based and satellite in situ observations have shown that in a climatological sense, the 
PRE plays a dominant role on the generation of equatorial $F$ region irregularities.

$\mathrm{F}$ region irregularities have been observed initiating at post-sunset hours in the equatorial region (Basu and Groves, 2001) and occurring at post-midnight hours, sometimes lasting till sunrise at mid-latitudes (Fukao et al., 1991). Under geomagnetically disturbed conditions, the density depletions could also be formed at mid-latitudes by penetrating electric field or disturbance electric field just as they are formed in the equatorial region ( $\mathrm{Li}$ et al., 2009). However, under geomagnetically quiet conditions, radar observations indicate that the mid-latitude $\mathrm{F}$ region irregularity structures are quite different from equatorial ones and might be associated with the mid-latitude instabilities (Kelley and Fukao, 1991). Based on the HF (high frequency) Doppler observations, Xiao et al. (2007) found that MSTIDs (mediumscale traveling ionospheric disturbances) generated during typhoons could trigger spread $\mathrm{F}$ at mid-latitudes. The relation between mid-latitude spread $\mathrm{F}$ and acoustic-gravity waves (AGWs) was verified later (Xiao et al., 2009). But the detailed mechanism still needs further investigation. In the case of low latitudes, the condition is rather complicated considering that both equatorial and mid-latitude instabilities could occur (Yokoyama et al., 2011a, b). Thus, the generation of post-midnight $\mathrm{F}$ region irregularities at low latitude has remained a research focus in recent years.

Using the Kototabang (dip lat. $10.4^{\circ} \mathrm{S}$ ) VHF (very high frequency) radar measurements, Otsuka and Ogawa (2009) classified the low-latitude $\mathrm{F}$ region irregularities into equinoctial pre-midnight ones and June solstitial postmidnight ones. The former ones are associated with equatorial plasma bubbles and the latter are of mid-latitude type which present much weaker echo intensity than their premidnight counterparts. Furthermore, Patra et al. (2009) found that the June solstitial post-midnight $\mathrm{F}$ region irregularities at Gadanki (dip lat. 6.4 $\mathrm{N}$ ) had Doppler velocities different from those of the decaying equinoctial post-midnight $\mathrm{F}$ region irregularities. They concluded that the equinoctial premidnight and June solstitial post-midnight irregularities are of different characteristics and should be investigated differently. The equinoctial pre-midnight irregularities maximize at equatorial latitudes while the June solstitial postmidnight ones maximize at middle latitudes. Specifically, using ionosonde ionogram measurements at equatorial latitudes in American, Pacific and Southeast Asian regions, Li et al. (2011) found that the F region irregularities during June solstice of solar minimum occurred from midnight to postmidnight hours with maximum occurrence rates of 50,80 and $30 \%$, respectively, but no marked TEC (total electron content) fast fluctuations were detected. They proposed that the June solstitial post-midnight $\mathrm{F}$ region irregularities at solar minimum might not cause GPS (Global Positioning System) scintillation. This, however, needs to be investigated further using simultaneous measurements from digital ionosondes and GPS scintillation receivers. Considering that irregulari- ties generated due to the equatorial plasma instability process or generated locally through mid-latitude instabilities can be observed at low latitudes, Fukao et al. (2004) elucidated that low latitudes represent an important area to investigate the irregularity generation mechanism of both equatorial- and mid-latitude types.

Sanya $\left(109.6^{\circ} \mathrm{E}, 18.3^{\circ} \mathrm{N}\right.$; dip lat. $\left.12.8^{\circ} \mathrm{N}\right)$ is situated at a low latitude in the Northern Hemisphere. Using the collocated VHF radar, digital ionosonde and GPS scintillation and TEC receiver at Sanya, Li et al. (2012) reported several cases of midnight backscatter plasma plumes without GPS scintillation. Hu et al. (2014) investigated the extent of height and duration of the $\mathrm{F}$ region irregularity echoes detected by the Sanya VHF radar. However, the report of Li et al. (2012) is only a case study and the report of $\mathrm{Hu}$ et al. (2014) focuses on the occurrence characteristics of radar echoes. A statistical comparative study of GPS scintillation and ionogram spread F, especially during June solstice, is still not performed. In this study, we investigate the local time and seasonal behavior of spread $\mathrm{F}$ and scintillations observed over Sanya. The analysis mainly focuses on (1) the statistical correlation between ionogram spread $\mathrm{F}$ and scintillation indices, and (2) the possible factors responsible for the occurrence of midnight/post-midnight spread $\mathrm{F}$ without scintillation.

\section{Data and methods}

Spread-F measurements were performed using the Digisonde Portable Sounder (DPS-4D) every $5 \mathrm{~min}$ at Sanya (Li et al., 2012). Using the SAO Explorer, the spread-F index, QF, which is defined as the mean range spread of the diffusing $\mathrm{F}$ layer trace, can be obtained directly from the ionograms by the Automatic Real-Time Ionogram Scaler with True height (ARTIST) (Galkin et al., 2008). The mean range spread refers to the averaged spread range of all frequencies detecting a diffuse echo in an ionogram. The spread range at each frequency is the virtual height difference between the top and bottom of the spread echo at the particular frequency (Abdu et al., 2012). The unit of QF is kilometers. If no spread $\mathrm{F}$ appears on the ionogram, the QF is assigned to NaN (not a number). The ionospheric Es layer critical frequency ( $f o E s$ ) used in the present study is manually scaled from the ionograms.

The GPS Ionospheric Scintillation and TEC Monitor (GISTM) system, GSV4004A, has been operating at the Sanya station since 2004. It is used to measure the ionospheric scintillations and slant total electron content (STEC). The amplitude scintillation index S4 related to density irregularities is defined as the standard deviation of signal intensity normalized to its average value (Briggs and Parkin, 1963) and is computed every $1 \mathrm{~min}$ based on a $50 \mathrm{~Hz}$ sampling rate. The GISTM computes S4T (the total scintillation index) and $\mathrm{S} 4 c$ (caused by ambient noise), so we get the $\mathrm{S} 4$ caused by irregularities in the form described in Van Dierendonck and 


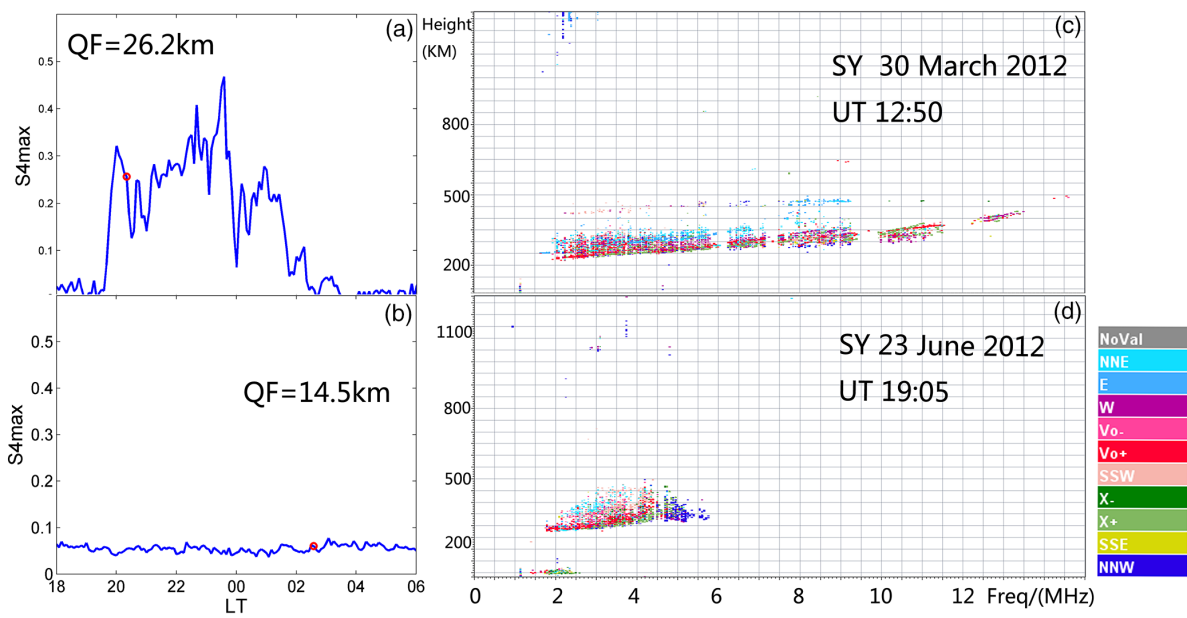

Figure 1. Examples of spread-F events $(\mathbf{a}, \mathbf{b})$ with and $(\mathbf{c}, \mathbf{d})$ without scintillations at Sanya. The red circles in panels (a) and (c) represent the time when the ionograms shown in right panels were measured. The corresponding QF index is also shown in the panel.

Hua (1998). The equation is

$\mathrm{S} 4=\sqrt{\mathrm{S} 4 T^{2}-\mathrm{S} 4 c^{2}}$.

In the present calculation, only data from satellites with elevation angles larger than $40^{\circ}$ and lock times longer than $180 \mathrm{~s}$ are used to reduce the multi-path effect. The $\mathrm{S} 4$ taken by all satellites within $1 \mathrm{~min}$ intervals are averaged. Since the temporal resolution of $\mathrm{S} 4$ and $\mathrm{QF}$ are 1 and $5 \mathrm{~min}$, respectively, we use the maximum S4 (S4max) in every 5 min to characterize the scintillation level and thus a 5 min resolution S4max sequence is generated.

Besides the S4 index, the STEC data are recorded by the GISTM. The appearance of possible MSTIDs can be detected in the TEC perturbations. In this study, a method similar to that used in Chen et al. (2011) is employed to get the TEC perturbations from original STEC observations. First, we select an observational sequence longer than $2 \mathrm{~h}$ and convert the STEC time series observed by each satellite-receiver pair into vertical TEC. Second, the $1.2 \mathrm{~h}$ running window averaged background TEC disturbances are extracted. However, after this procedure, we found that the resultant TEC variations were still not absolutely detrended. Therefore, we further remove the quadratic fit in the $1.2 \mathrm{~h}$ running window from the resultant temporal VTEC (vertical total electron content) variation obtained in last procedure and thus get the VTEC perturbation sequence. A possible MSTID event is manually identified as a sequence with at least two wave structures, of 15-60 min period, and 0.4 TECU (TEC units) fluctuation amplitudes. In selecting the amplitude limitation for the possible MSTID, we refer to a previous study by Chen et al. (2011) that used fluctuations with amplitudes larger than $1 \%$ of the background TEC to determine if MSTID occurred or not. Ding (2011) found that the amplitudes of nighttime MSTIDs at Wuhan, which is situated at a similar longitude as Sanya, were $2-7 \%$. The average nighttime back- ground TEC in June solstice 2012 over Sanya is 23 TECU (as derived from the global ionospheric maps). According to the research conducted by Ding et al. (2011), the lowest amplitude of the nighttime MSTIDs was $2 \%$ of the background value of 23 TECU, i.e., 0.46 TECU. We take 0.4 TECU as the threshold in our study.

\section{Results}

A case study of spread $\mathrm{F}$ and scintillations has shown two kinds of irregularities that appeared during equinox and June solstice, respectively (Li et al., 2012). In our study, both types are statistically investigated through analyzing the data collected from the GPS scintillation and TEC receiver, and the digital ionosonde at Sanya. Figure 1a and b show a severe spread-F event accompanied by strong scintillation that began around 20:00 LT (LT $=\mathrm{UT}+7.5 \mathrm{~h}$ at Sanya) on 30 March 2012. The scintillation lasts for nearly $5 \mathrm{~h}$ and the peak of S4max is $\sim 0.5$. A typical ionogram representing strong range-type spread $\mathrm{F}$ is shown in Fig. 1b. The corresponding QF and S4 indices (marked as a red circle in Fig. 1a) are $26.4 \mathrm{~km}$ and 0.25 , respectively. On the other hand, Fig. 1c and d show an event that occurred postmidnight on 23 June 2012. The event is significantly different from the equinoctial one shown in Fig. 1a and b. The spread $\mathrm{F}$ belongs to the mixed type (with both range and frequency spread F) and no gigahertz $(\mathrm{GHz})$ scintillation was found. The June solstitial post-midnight irregularities without causing GPS scintillations were also reported in earlier radar observations over Kototabang (Otsuka and Ogawa, 2009).

To obtain the statistical behavior of S4 and QF, we investigate the local time and seasonal distributions of $\mathrm{S} 4 \mathrm{max}$ and QF during the period December 2011-November 2012. The time resolution is $5 \mathrm{~min}$. Considering that irregularities occur mostly after sunset, we only present data from 18:00 to 07:30 LT. The light gray areas in Fig. 2a and b represent the 

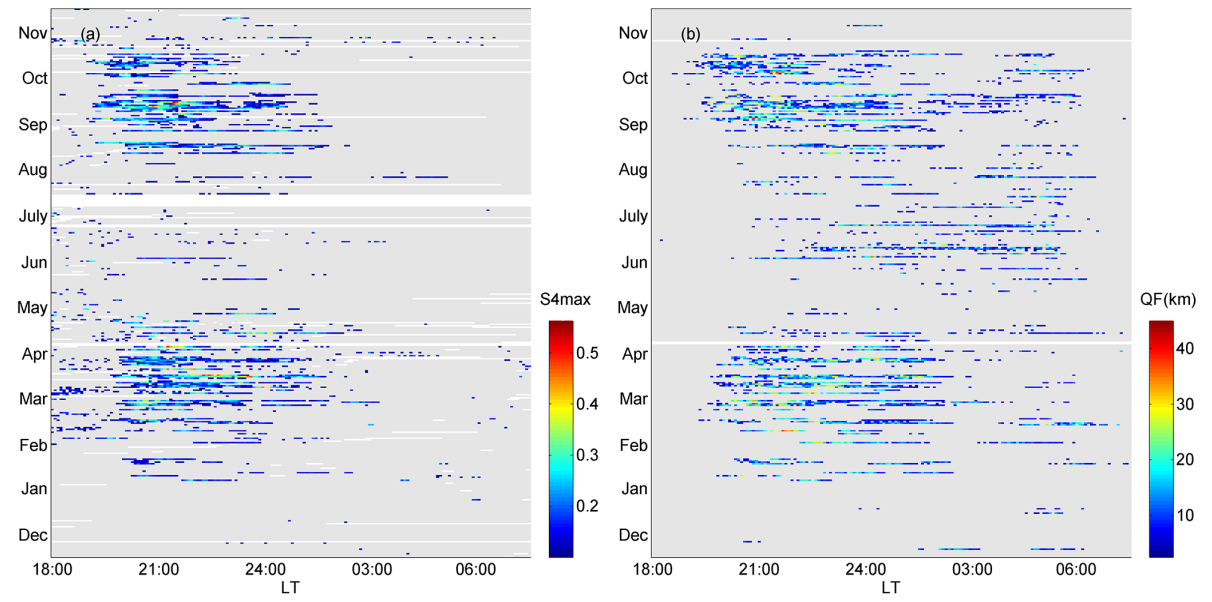

Figure 2. Seasonal and local time (LT) variations of (a) S4max and (b) QF indices observed over Sanya during December 2011-November 2012.
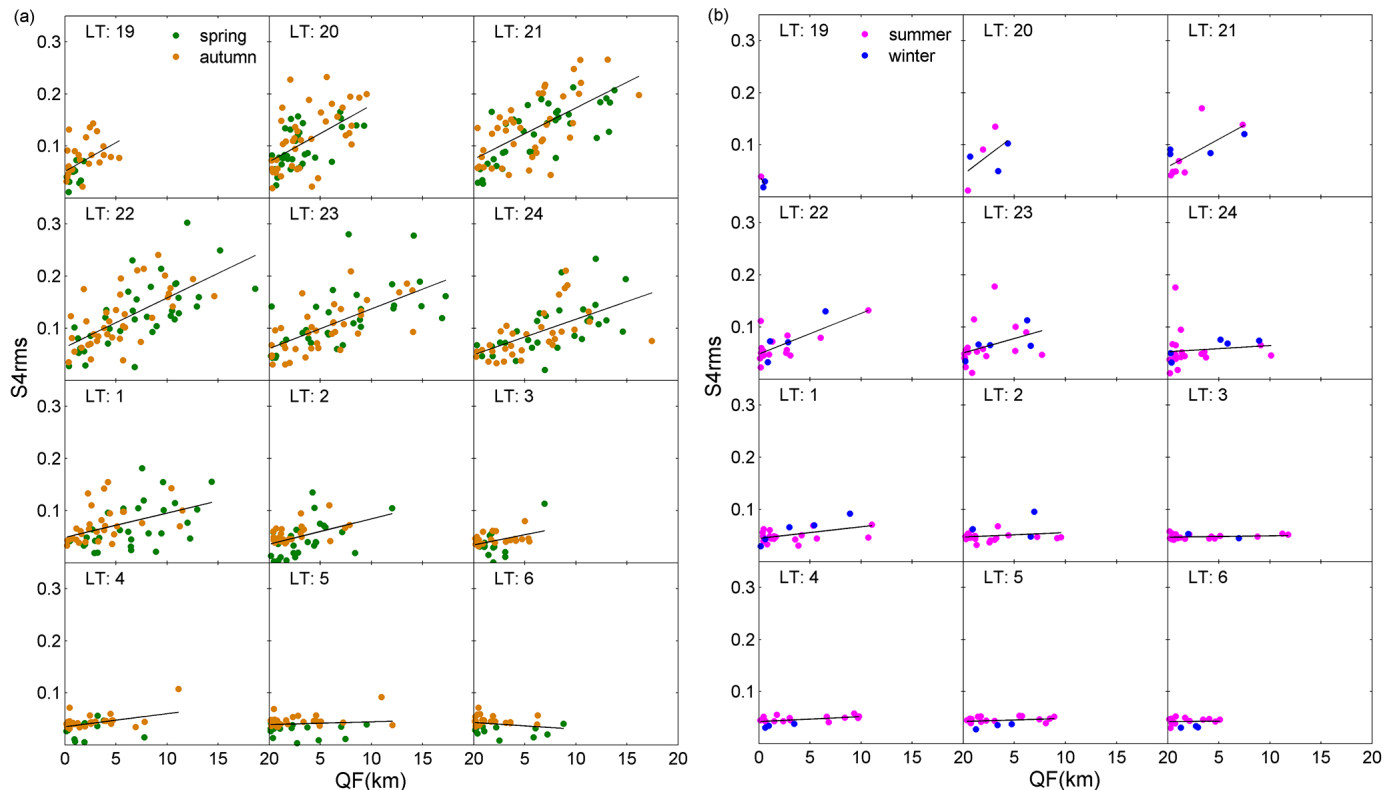

Figure 3. Scatter plots of $1 \mathrm{~h}$ averaged QF (QFavg) and root mean square of S4max (S4rms) during (a) equinoctial and (b) solstitial months. The solid slant line in each plot is the linear fit of all data.

periods when S4max was less than 0.1 and no spread $F$ was detected (as presented by QF), respectively. The absence of data due to equipment failure is represented in white color in both figures. It can be seen from Fig. 2a that S4max is large after sunset around equinox but is low in the post-midnight period. No apparent scintillation activity was detected during June solstice. The results are similar to those observed around the equatorial ionization anomaly. By analyzing the GPS L-band scintillation data obtained at Guangzhou and Shenzhen from July 2008 to March 2012, Deng et al. (2013) found that scintillation occurred only in the pre-midnight period at equinox during low solar activity years. In the case of the QF index, Fig. 2b shows that it is strong in the post- sunset period in equinox (similar to S4max) and moderate post-midnight in June solstice (different from the absence of scintillation). The different behaviors of S4max and QF indices indicate different evolutions of $400 \mathrm{~m}$ and severalkilometers-scale irregularities during different seasons.

Figure $3 \mathrm{a}$ and $\mathrm{b}$ show the scatter plots of S4max and QF as a function of local time during equinoctial and solstitial months, respectively. The resolution of the data is processed into $1 \mathrm{~h}$ using the following method. The mean QF (QFavg) and the root mean square of S4max (S4rms) are calculated in a $2 \mathrm{~h}$ running window, and the data centered on the hour are extracted from the $5 \mathrm{~min}$ resolution sequence; the resulting series are of $1 \mathrm{~h}$ resolution. The data in spring (February- 

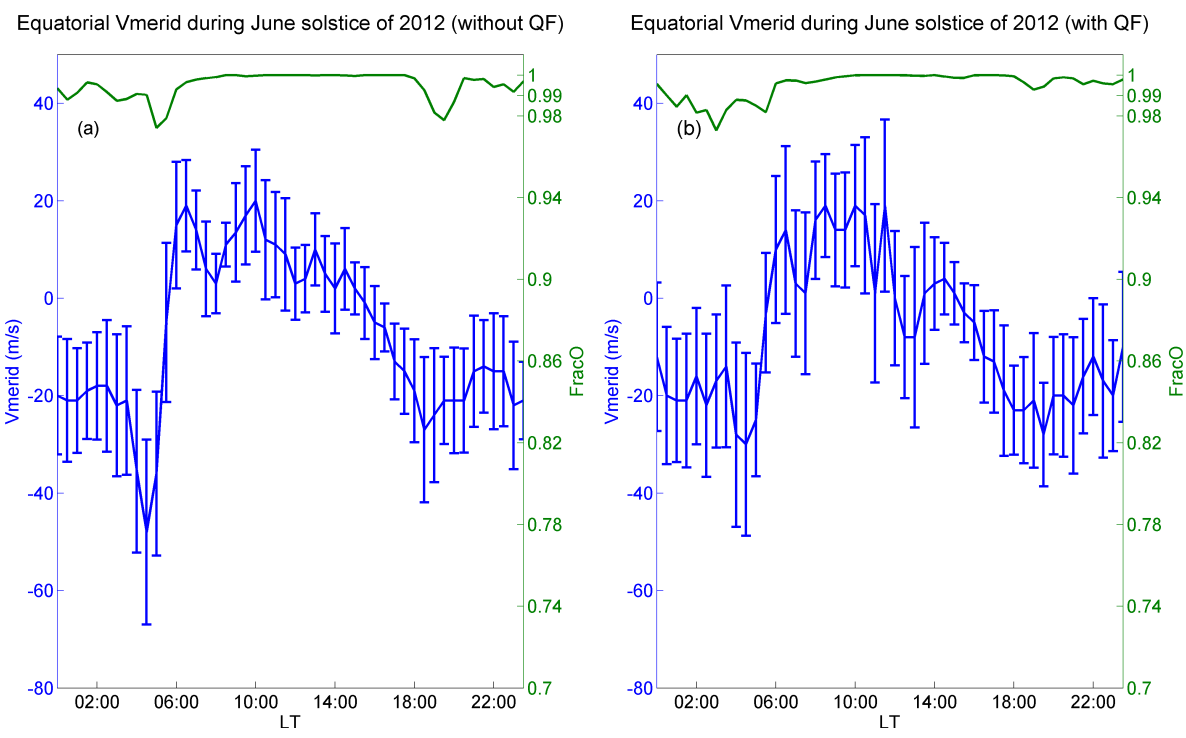

Figure 4. The median equatorial Vmerid (vertical drift velocity) and fraction of $\mathrm{O}^{+}$obtained from C/NOFS during (a) non-QF days and (b) QF days in June solstice (May-August 2012) at $102-116^{\circ} \mathrm{E}$ longitude and $5^{\circ} \mathrm{S}-5^{\circ} \mathrm{N}$ magnetic latitude.

April), summer (May-July), autumn (August-October) and winter (November-January) are represented by green, pink, orange and blue dots, respectively. The straight line in each plot is the linear fit of all data. Figure 3a shows that S4rms increases with QFavg during equinox. The slope is largest from 19:00 to 24:00 LT. This might be attributed to the fact that different scale irregularities producing apparent QFavg and S4rms coexist in the pre-midnight periods in equinoctial months. Afterwards, the QFavg and S4rms decrease and the slope becomes small indicating the decay of the irregularities. Regarding solstitial data shown in Fig. 3b, both indices are smaller than that at equinox and S4rms is mostly less than 0.1. S4rms shows a little decreasing trend from 21:00 to 05:00 LT, while QF remains moderate even after midnight. The possible factors responsible for the differences of the variation patterns of QF and S4 (Fig. 3a, b) will be discussed in the following section.

\section{Discussion}

Case and statistical analyses show that the irregularities observed during equinox and June solstice present distinct local time distribution patterns. Equinoctial irregularities relating to equatorial plasma bubbles (EPBs) (Li et al., 2012) are dominated in the post-sunset hours. In post-sunset periods, the Rayleigh-Taylor instability is usually triggered at the bottom side of a rising equatorial $F$ layer. The growth rate of instability is mainly affected by meridional wind and prereversal enhancement of the eastward electric field (PRE) determined by the longitudinal gradient of flux-tube-integrated conductivity. Of all these factors, PRE is the dominant one. Considering that the magnetic field line is nearly aligned with the solar terminator in equinox at Sanya longitude, the PRE would be largest in this season. Larger PRE tends to trigger more severe plasma depletion (Su et al., 2008; Chapagain et al., 2009). In this situation, the equatorial field-aligned density depletions, ascending through the $\mathrm{F}$ region to higher altitudes, map along the magnetic field line to low latitude ( $\mathrm{Li}$ et al., 2013). Multi-scale irregularities, ranging from a few kilometers responsible for the $\mathrm{QF}$ to several hundred meters that produce the $\mathrm{GHz}$ scintillations, coexist in equatorial depletions (Basu et al., 1978). Therefore, QF and S4max are both large during equinoctial post-sunset hours.

In June solstice, spread-F irregularities do not appear until late at night. The absence of post-sunset irregularities can be attributed to the small PRE. In addition, the irregularities during midnight/post-midnight hours are more intense than during late post-sunset hours; therefore, the post-midnight irregularities could not be a continuation of the post-sunset irregularities such as those observed during the equinox. The vertical drift is known as one of the most important factors for the generation of equatorial spread F. It was found that the correlation coefficient between the EPB occurrence probability and the magnitude of the PRE during quiet time was as high as 0.75 (Kil et al., 2009). To investigate the possible effect of vertical drift on the generation of June solstitial post-midnight irregularities observed over Sanya, we analyze the median meridional (vertical) ion drift velocity, Vmerid, obtained from C/NOFS (Communication Navigation Outage Forecasting System) during the period from May to July in the area $\left(102-116^{\circ} \mathrm{E}\right.$ in longitudes and $5^{\circ} \mathrm{S}-5^{\circ} \mathrm{N}$ in magnetic latitudes). The drift data are obtained under the condition that the fraction of $\mathrm{O}^{+}$to the total ion concentration is larger than 0.85 and that the $\mathrm{O}^{+}$concentration is larger than $3 \times 10^{3} \mathrm{~cm}^{-3}$ to ensure reliability for each data point. We divide the data into two groups: one comprises data taken on 

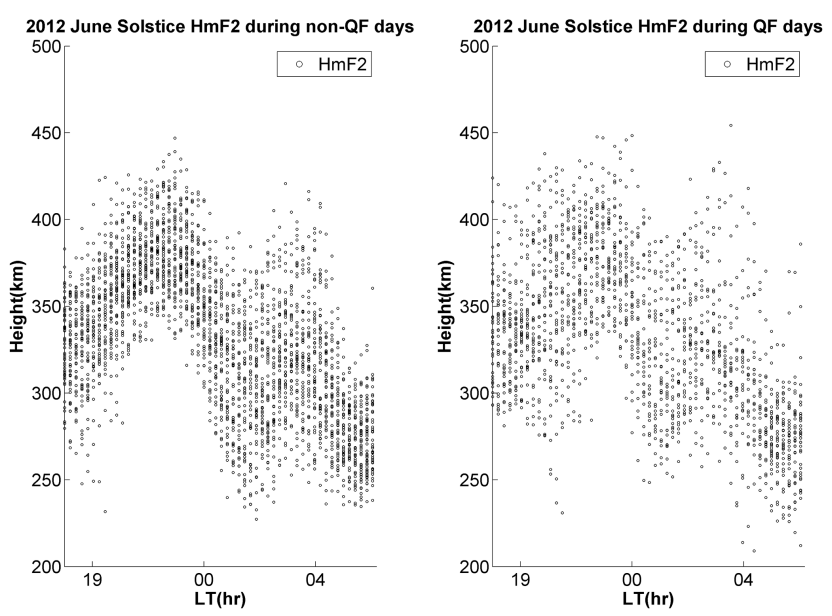

Figure 5. Local time variations of $F$ layer peak height over Sanya during June solstitial non-QF days (left) and QF days (right).

quiet non-QF days and the other on quiet QF days. As shown in Fig. $4 \mathrm{a}$ and $\mathrm{b}$, the peaks of Vmerid are 20 and $15 \mathrm{~m} \mathrm{~s}^{-1}$ downward during non-QF and QF days, respectively. At midnight, under both conditions, the fraction of $\mathrm{O}^{+}$is close to 1 , which indicates the credibility of the drift data. In order to figure out whether the vertical plasma motion plays a role on the generation of June solstitial irregularities over Sanya, we examine the June solstitial F2 layer peak height ( $h m \mathrm{~F} 2)$ measured by the Sanya ionosonde during non-QF and QF days. As can be seen in Fig. 5, the F layer peak heights under both conditions have a peak around 22:00 LT. Referring to the vertical drift obtained by C/NOFS in Fig. 4a, there is a decrease of downward drift around 21:00-22:00 LT. It is concluded that the height rise observed by ionosonde over Sanya might be a manifestation of a decreasing downward drift together with sufficient midnight chemical recombination rate and horizontal plasma flux (Nicolls et al., 2006). During equinox, when F layer uplift is known to be an important contributor to the generation of post-sunset irregularities, the statistical behavior of post-sunset $\mathrm{F}$ layer peak height during QF days is different from that during non-QF days. On the other hand, the June solstitial F layer peak heights do not manifest a statistical difference between QF days and non-QF days. However, by examining the June solstitial data, we found that most irregularities start after 22:00 LT when the $\mathrm{F}$ layer heights reach the peak. Therefore, the midnight height increase of the $\mathrm{F}$ layer might provide a favorable condition for the generation of June solstitial spread F, but it is not a conclusive contributor to decide whether irregularities occur or not on a given day.

Differing from the large QF and S4max at equinox, the QF events observed at June solstice were rarely accompanied by scintillations. Whalen (2009) reported that if the background density is denser, the amplitude of scintillation tends to be larger. If the irregularities intersect in an extremely low background density, for example in the post-midnight period dur- ing June solstitial months of a low solar activity year when the background electron density is very low (Liu et al., 2011), the scintillation can hardly be detected. On the other hand, we classify the spread-F events as range type, frequency type and a mixture of range and frequency type. During June solstice, $57 \%$ of the spread-F events observed over Sanya belong to the mixture type. In general, the mixed-type spread $\mathrm{F}$ has two sources: it drifted from elsewhere or was generated locally over Sanya. For the spread F drifted from elsewhere, it might have been range type during its initial period and then evolved into mixed-type spread $\mathrm{F}$ when it drifted over Sanya. The simultaneous observations from the Sanya VHF radar and ionosonde show that the mixed-type spread $\mathrm{F}$ during equinoctial months are generated in the western longitudes of Sanya (Li et al., 2012). The equinoctial mixed-type spread F usually causes strong GPS scintillations. However, for the present June solstitial mixed-type spread F observed over Sanya, no simultaneous GPS scintillation was detected. The observation indicates that the June solstitial mixed-type spread $\mathrm{F}$ is different from that observed during equinoctial months over Sanya. Chakraborty et al. (2012) reported that the mid-latitude spread $\mathrm{F}$ was mostly of frequency or mixedtype, which could cause VHF scintillation but no GHz scintillation during magnetically disturbed time (Ledvina et al., 2002; Doherty et al., 2003). Using simultaneous rocket and ionosonde $\left(37.95^{\circ} \mathrm{N}, 284.53^{\circ} \mathrm{E}\right.$, dip lat. $\left.48.5^{\circ} \mathrm{N}\right)$ measurements, Earle et al. (2010) found that the ionosonde detected a mix of range and frequency type spread $\mathrm{F}$ during the flight of the rocket, but no significant plasma density structures smaller than a few kilometers were observed in the rocket data. This indicates that the mid-latitude mixed-type spread F only comprises kilometer-scale (without $400 \mathrm{~m}$ ) irregularities.

On the basis of the simultaneous observations of spread $\mathrm{F}$ and GPS scintillations over Sanya, the June solstitial mixedtype spread F could belong to the middle-latitude-type spread F which cannot cause the $\mathrm{GHz}$ scintillation; unlike the equatorial ones which can cause the $\mathrm{GHz}$ scintillation, the failure of detection of the $\mathrm{GHz}$ scintillation is most likely caused by the effect of low background electron density. For the generation of mid-latitude-type irregularities, Perkins instability is considered as the most probable mechanism. But the growth rate of Perkins instability is not large enough to explain observational results. Cosgrove (2007) proposed that the combined system of $\mathrm{E}$ and $\mathrm{F}$ layers has to be considered, which is supported by the fact that the coupled Perkins instability and Es layer instability have larger growth rates than by either one alone (Cosgrove and Tsunoda, 2004). This result has also been reproduced through a simulation study (Yokoyama et al., 2009). The electric field with scales $>10 \mathrm{~km}$ can be mapped very efficiently between the $\mathrm{E}$ and $\mathrm{F}$ layers. Tsunoda and Cosgrove (2001) suggested that there is a large feedback polarization electric field mapped from the $\mathrm{E}$ layer and contributing to the $\mathrm{F}$ region irregularities. To validate the linkage between spread F and Es, Haldoupis et al. (2003) investi- 

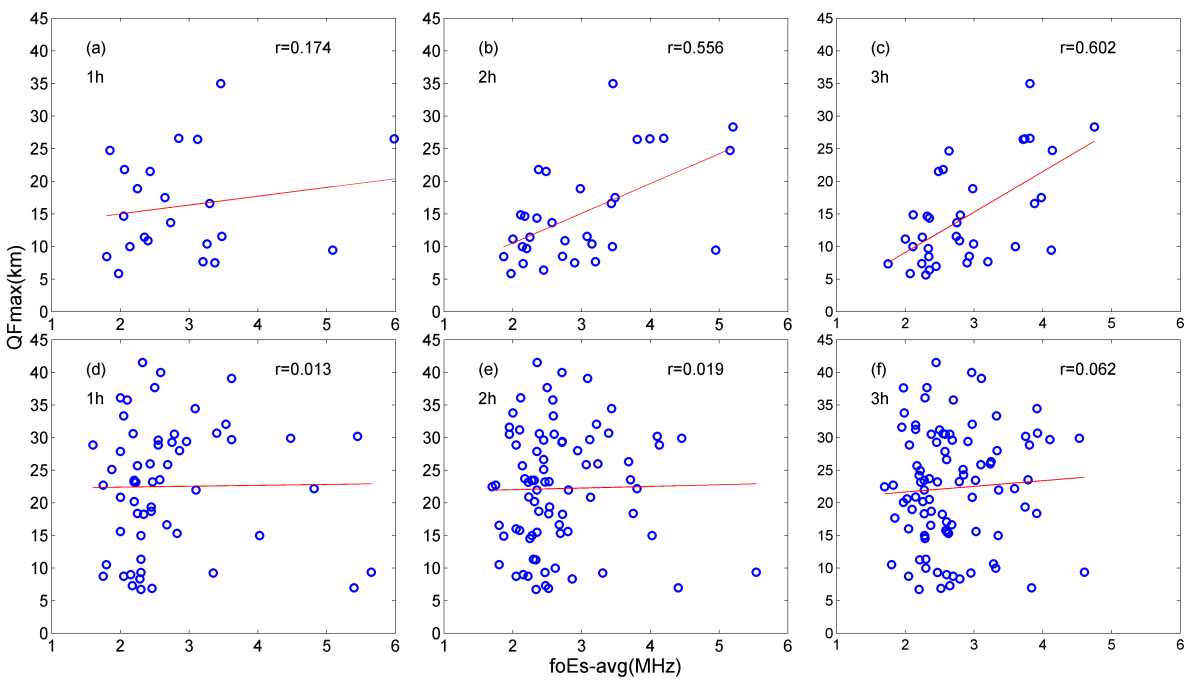

Figure 6. Scatter plots of maximum QF (QFmax) and average of $f o$ Es ( $f o$ Es-avg) in 1 (a, d), 2 (b, e) and 3 (c, f) hours before QF events under quiet conditions (the sum of daily $\left.\mathrm{Kp}, \sum \mathrm{Kp}<24\right)$ during $(\mathbf{a}-\mathbf{c})$ June solstice and $(\mathbf{d}-\mathbf{f})$ equinox. The red line in each panel is the fitting of all data. The correlation coefficient is shown in the right top of each panel.

gated recordings of ionosonde in summer and found a precise corresponding relation between spread $\mathrm{F}$ and the patchy, sporadic E layer. They concluded that the enhanced electric field set up in unstable Es could easily map up to $\mathrm{F}$ region and trigger gradient drift instability to form mid-latitude irregularities. This procedure is considered to occur at low latitudes as well. Based on joint observations from the MST (mesosphere-stratosphere-troposphere) radar and ionosonde at a low-latitude station, Gadanki, Patra et al. (2009) proposed that the Es layer might be the energy source of Rayleigh-Taylor and gradient drift instabilities producing the post-midnight field-aligned irregularities (FAIs). While considering the Es and its associated irregularities, Ning et al. (2012) and Li et al. (2014) reported a high occurrence of E region irregularities during June solstice with the Sanya VHF radar observations. Thus, the Es could possibly contribute to the generation of the post-midnight spread $\mathrm{F}$ over Sanya.

A one-to-one correlation between spread $\mathrm{F}$ and Es in June solstice $(\mathrm{a}-\mathrm{c})$ and equinox $(\mathrm{d}-\mathrm{f})$ is presented in Fig. 6. Maximum QF (QFmax) and average of $f o$ Es ( $f o$ Es-avg) are taken as the indicators of the intensities of spread $\mathrm{F}$ and Es activity, respectively. The red line in each panel is the fitting of all data and the correlation coefficient is shown in the right top of each panel. The equinoctial correlation (Fig. 6d-f) is extremely low. Through examination of the QF events in equinox 2012, we find that 104 events occurred at equinox, 67 of them have Es events, but 100 of them occurred before 23:00 LT. No Es events were detected $3 \mathrm{~h}$ before the other four QF events which occurred after 23:00 LT. It seems that Es has no effect on the freshly generated equinoctial postmidnight irregularities. On the other hand, Fig. $6 \mathrm{~b}$ and $\mathrm{c}$ show moderate correlations, 0.556 and 0.602 , respectively. This might indicate that the summer solstitial Es activities play a role in the generation of spread-F irregularities. However, it is noted that the correlation in Fig. 6a is low. Through manually checking ionograms we found that during weak spread-F conditions, no QF values can be obtained from the SAO Explorer. This could produce a statistical bias in the estimate of time delay between the Es and spread-F occurrence. A statistical analysis on the onset time of spread F and QF shows that the time interval between them ranges from 0 to $60 \mathrm{~min}$. Therefore, the time delay between the appearance of Es and QF does not necessarily represent the time required for the establishment of the instability that causes the spread-F development. In this regard, Yokoyama et al. (2009) reported that during the most favorable condition, the coupled EsPerkins instability takes $700 \mathrm{~s}(\sim 12 \mathrm{~min})$ to generate variations sufficient to account for the observed airglow and GPSTEC perturbations by Saito et al. (2001). Thus, the foEs averaged during a $1 \mathrm{~h}$ interval before QF may have little correlation with $\mathrm{QF}$.

Besides the Es, MSTID is proposed as another possible contributor to the generation of June solstitial post-midnight irregularities. Observational data of TEC fluctuations and spread $\mathrm{F}$ over Wuhan $\left(30.5^{\circ} \mathrm{N}, 114.4^{\circ} \mathrm{E}\right)$ present a consistency of seasonal variations between MSTIDs and spread F (Chen et al., 2011). Sanya and Wuhan are situated at nearby longitudes, while the latitude of Sanya is lower by about $10^{\circ}$. C/NOFS satellites revealed the existence of MEF (midlatitude electric field fluctuation) associated with MSTIDs at latitudes as low as $10^{\circ} \mathrm{S}$ (dip latitude) (Yokoyama et al., 2011a). Based on this result, since Sanya is located at a similar latitude, it is possible that MSTIDs contribute to the generation of irregularities over Sanya. In a case study made at the Sanya station, a wave-like structure of F2 peak height was 


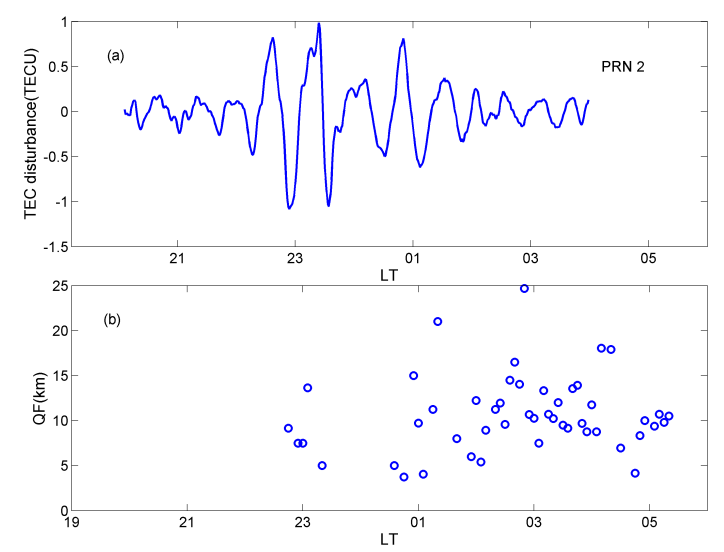

Figure 7. Local time variations of (a) TEC perturbations and of (b) QF obtained on 23 June 2012 over Sanya.

found in a rising $\mathrm{F}$ layer before the spread-F event. It was speculated to be a signature of TIDs (traveling ionospheric disturbances) or gravity waves (Li et al., 2012). According to previous studies, the propagation direction of MSTID is very important (Shiokawa et al., 2003b). However, due to the fact that only one GPS scintillation and TEC receiver is operated around Sanya, it is difficult to get the propagation direction based on our data. Referring to previous studies, Shiokawa (2003a) observed the MSTIDs over Shigaraki $\left(34.9^{\circ} \mathrm{N}, 25.4^{\circ}\right.$ magnetic latitude) and found that most of the MSTIDs propagate southwestward in airglow images. According to a previous study conducted at a similar longitude to Sanya's, nighttime MSTIDs tend to propagate southwestward in the Northern Hemisphere, regardless of season or latitude (Ding et al., 2011). The middle-latitude electric field (MEF) associated with MSTID has been observed at $10.36^{\circ} \mathrm{S}$ dip latitude (Yokoyama et al., 2011a), which is similar to the latitude of Sanya. The MSTIDs propagating southwestward are believed to originate from middle latitudes (Shiokawa et al., 2003b; Otsuka et al., 2004). The polarization electric field inside a MSTID might contribute to the generation of irregularities through $\boldsymbol{E} \times \boldsymbol{B}$ instability (Kelley and Fukao, 1991). In our study, we extract possible MSTIDs from TEC observations using the method described in Sect. 2. One example of possible MSTID sequence observed by PRN2 on 23 June 2012 is shown in Fig. 7a. It lasts for nearly $4 \mathrm{~h}$ and the fluctuation amplitude is as large as 1 TECU. Figure 7b shows QF variation on that day. The QF indices were obtained automatically from the ionograms by ARTIST. It is noted that the QF event occurred half an hour after the appearance of possible MSTIDs. Similar to the time delay between Es and QF, this time delay is also affected by the failure of obtaining QF by the SAO Explore under weak spread$F$ conditions, and it does not necessarily represent the time required for the establishment of the instability that causes the spread-F development. It is reported that the growth rate of Perkins instabilities associated with MSTIDs is low. But

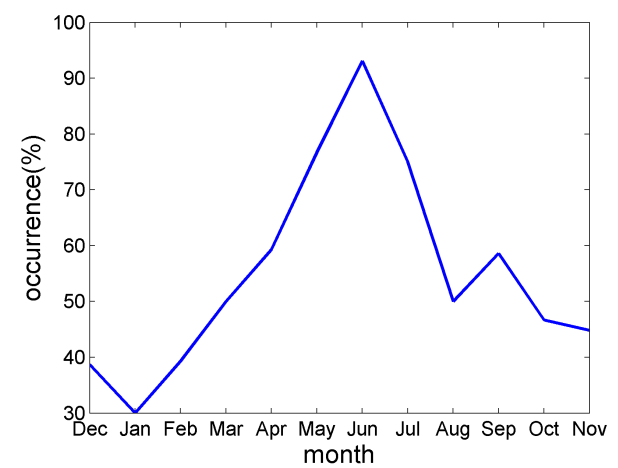

Figure 8. Monthly nocturnal occurrence rates of possible MSTID activity over Sanya during December 2011-November 2012 under geomagnetically quiet conditions $\left(\sum \mathrm{Kp}<24\right)$.

if it combined with gravity wave seeding or Es layer instabilities, the growth time would be reduced (Kelley and Fukao, 1991).

In order to further investigate the relation between spread $\mathrm{F}$ and possible MSTIDs, the monthly nocturnal occurrence rates of possible MSTID activity under geomagnetically quiet periods (the sum of daily $\mathrm{Kp}<24$ ) are presented in Fig. 8. The statistical data were selected using the same method as in Fig. 7, and only data from 19:00 to 04:00 LT and under geomagnetically quiet periods were used to avoid the impacts from sunrise/sunset and magnetic storms. The monthly occurrence rate is defined as the number of days with at least one MSTID event divided by the number of days with proper observations in a month. Figure 8 shows that MSTID activities prefer to occur in June solstice. The peak occurrence in June is as high as $93 \%$. Occurrences from May to September are all over 50\%. Furthermore, comparing the local time distribution of QF shown in Fig. 1b with that of possible MSTIDs, we find that they are distributed in similar local time windows. By analyzing the data in detail, we find there are $40 \mathrm{QF}$ events occurring during June solstice; 32 of them have possible MSTIDs detected within $3 \mathrm{~h}$ before the QF events occur. All of these contribute to the speculation that MSTIDs might play a role in the production of June solstitial post-midnight irregularities over Sanya.

\section{Conclusions}

In this paper, we presented a comparative study of spread $\mathrm{F}$ and scintillation through QF and S4 indices recorded by a digital ionosonde and a GPS scintillation and TEC receiver, respectively, at Sanya during December 2011-November 2012. Distinct differences between irregularities observed during equinox and June solstice have been found. During equinoctial months, the QF and S4max indices are both large and S4max increases with QF in the pre-midnight period. Then both of them, especially S4max, decrease due to the fast decay of $400 \mathrm{~m}$ scale irregularities. In the case of the 
June solstice, moderate QF appears late at night; no corresponding scintillation is found. The generation of June solstitial post-midnight irregularities is suggested to be related to possible MSTIDs and Es activity frequently observed at the Sanya station.

Acknowledgements. This work is supported by the Chinese Academy of Sciences (KZZD-EW-01-2), National Important Basic Research Project (2011CB811405), and National Science Foundation of China (41321003, 41131066, 41427901, 41422404). We acknowledge the use of data from the Chinese Meridian Project.

The topical editor H. Kil thanks M. Abdu and one anonymous referee for help in evaluating this paper.

\section{References}

Abdu, M., Batista, I., and Bittencourt, J.: Some characteristics of spread-F at the magnetic equatorial station Fortaleza, J. Geophys. Res., 86, 6836-6842, 1981.

Abdu, M., Batista, I., Reinisch, B., MacDougall, J., Kherani, E., and Sobral, J.: Equatorial range spread-F echoes from coherent backscatter, and irregularity growth processes, from conjugate point digital ionograms, Radio Sci., 47, RS6003, doi:10.1029/2012RS005002, 2012.

Abdu, M. A.: Outstanding problems in the equatorial ionospherethermosphere electrodynamics relevant to spread-F, J. Atmos. Sol.-Terr. Phy., 63, 869-884, doi:10.1016/s13646826(00)00201-7, 2001.

Basu, S. and Groves, K.: Specification and forecasting of outages on satellite communication and navigation systems, Geoph. Monog. Series, 125, 423-430, 2001.

Basu, S., Basu, S., Aarons, J., McClure, J., and Cousins, M.: On the coexistence of kilometer-and meter-scale irregularities in the nighttime equatorial F region, J. Geophys. Res., 83, 4219-4226, 1978.

Briggs, B. and Parkin, I.: On the variation of radio star and satellite scintillations with zenith angle, J. Atmos. Terr. Phys., 25, 339366, 1963.

Burke, W. J., Huang, C. Y., Gentile, L. C., and Bauer, L.: Seasonallongitudinal variability of equatorial plasma bubbles, Ann. Geophys., 22, 3089-3098, doi:10.5194/angeo-22-3089-2004, 2004.

Chakraborty, S. K., Hajra, R., and DasGupta, A.: Ionospheric scintillation near the anomaly crest in relation to the variability of ambient ionization, Radio Sci., 47, RS2006, doi:10.1029/2011rs004942, 2012.

Chapagain, N. P., Fejer, B. G., and Chau, J. L.: Climatology of postsunset equatorial spread-F over Jicamarca, J. Geophys. Res., 114, A07307, doi:10.1029/2008JA013911 2009.

Chen, W., Lee, C., Chu, F., and Su, S.: Spread F, GPS phase fluctuations, and medium-scale traveling ionospheric disturbances over Wuhan during solar maximum, J. Atmos. Sol.-Terr. Phy., 73, 528-533, doi:10.1016/j.jastp.2010.11.012, 2011.

Cosgrove, R. B.: Generation of mesoscale $F$ layer structure and electric fields by the combined Perkins and $E_{S}$ layer instabilities, in simulations, Ann. Geophys., 25, 1579-1601, doi:10.5194/angeo-25-1579-2007, 2007.
Cosgrove, R. B. and Tsunoda, R. T.: Instability of the E-F coupled nighttime midlatitude ionosphere, J. Geophys. Res., 109, A04305, doi:10.1029/2003ja010243, 2004.

Deng, B., Huang, J., Liu, W., Xu, J., and Huang, L.: GPS scintillation and TEC depletion near the northern crest of equatorial anomaly over South China, Adv. Space Res., 51, 356-365, doi:10.1016/j.asr.2012.09.008, 2013.

Ding, F., Wan, W., Xu, G., Yu, T., Yang, G., and Wang, J.-S.: Climatology of medium-scale traveling ionospheric disturbances observed by a GPS network in central China, J. Geophys. Res., 116, A09327, doi:10.1029/2011JA016545, 2011.

Doherty, P. H., Delay, S. H., Valladares, C. E., and Klobuchar, J. A.: Ionospheric scintillation effects on GPS in the equatorial and auroral regions, 4, Institute of Navigation, Manassas, VA, USA, 11 pp., 2003.

Earle, G. D., Bhaneja, P., Roddy, P. A., Swenson, C. M., Barjatya, A., Bishop, R. L., Bullett, T. W., Crowley, G., Redmon, R., Groves, K., Cosgrove, R., and Vadas, S. L.: A comprehensive rocket and radar study of midlatitude spread-F, J. Geophys. Res., 115, A12339, doi:10.1029/2010JA015503, 2010.

Fukao, S., Kelley, M. C., Shirakawa, T., Takami, T., Yamamoto, M., Tsuda, T., and Kato, S.: Turbulent upwelling of the mid-latitude ionosphere: 1. Observational results by the MU radar, J. Geophys. Res., 96, 3725-3746, 1991.

Fukao, S., Ozawa, Y., Yokoyama, T., Yamamoto, M., and Tsunoda, R.: First observations of the spatial structure of $F$ region 3-m-scale field-aligned irregularities with the Equatorial Atmosphere Radar in Indonesia, J. Geophys. Res., 109, A02304, doi:10.1029/2003JA010096, 2004.

Galkin, I. A., Khmyrov, G. M., Kozlov, A. V., Reinisch, B. W., Huang, X., and Paznukhov, V. V.: The ARTIST 5, AIP Conf. Proc., 974, 150-159, doi:10.1063/1.2885024, 2008.

Haldoupis, C., Kelley, M. C., Hussey, G. C., and Shalimov, S.: Role of unstable sporadic-E layers in the generation of midlatitude spread-F, J. Geophys. Res., 108, doi:10.1029/2003JA009956, 2003.

Hu, L., Ning, B., Li, G., and Li, M.: Observations on the fieldaligned irregularities using Sanya VHF radar: 4. June solstitial $F$ region echoes in solar minimum, Chinese J. Geophys.-Ch., 57, 1-9, doi:10.6038/cjg20140101, 2014.

Hysell, D. L. and Burcham, J.: JULIA radar studies of equatorial spread-F, J. Geophys. Res., 103, 29155-29167, 1998.

Kelley, M. C. and Fukao, S.: Turbulent upwelling of the midlatitude ionosphere: 2. Theoretical framework, J. Geophys. Res., 96, 3747-3753, 1991.

Kil, H., Paxton, L. J., and Oh, S. J.: Global bubble distribution seen from ROCSAT-1 and its association with the evening prereversal enhancement, J. Geophys. Res., 114, A06307, doi:10.1029/2008JA013672, 2009.

Ledvina, B. M., Makela, J. J., and Kintner, P. M.: First observations of intense GPS L1 amplitude scintillations at midlatitude, Geophys. Res. Lett., 29, 4-1-4-4, doi:10.1029/2002GL014770, 2002.

Li, G., Ning, B., Liu, L., Zhao, B., Yue, X., Su, S. Y., and Venkatraman, S.: Correlative study of plasma bubbles, evening equatorial ionization anomaly, and equatorial prereversal $\mathrm{E} \times \mathrm{B}$ drifts at solar maximum, Radio Sci., 43, RS4005, doi:10.1029/2007RS003760, 2008. 
Li, G., Ning, B., Abdu, M., Yue, X., Liu, L., Wan, W., and Hu, L.: On the occurrence of postmidnight equatorial $\mathrm{F}$ region irregularities during the June solstice, J. Geophys. Res., 116, A04318, doi:10.1029/2010JA016056, 2011.

Li, G., Ning, B., Abdu, M. A., Wan, W., and Hu, L.: Precursor signatures and evolution of post-sunset equatorial spreadF observed over Sanya, J. Geophys. Res., 117, A08321, doi:10.1029/2012ja017820, 2012.

Li, G., Ning, B., and Hu, L.: Interferometry observations of lowlatitude E-region irregularity patches using the Sanya VHF radar, Sci. China Ser. A, 57, 1552-1561, doi:10.1007/s11431-0145592-3, 2014.

Li, G. Z., Ning, B. Q., Zhao, B. Q., Liu, L. B., Wan, W. X., Ding, F., Xu, J. S., Liu, J. Y., and Yumoto, K.: Characterizing the 10 November 2004 storm-time middle-latitude plasma bubble event in Southeast Asia using multi-instrument observations, J. Geophys. Res., 114, A07304, doi:10.1029/2009ja014057, 2009.

Li, G. Z., Ning, B. Q., Abdu, M. A., Otsuka, Y., Yokoyama, T., Yamamoto, M., and Liu, L. B.: Longitudinal characteristics of spread-F backscatter plumes observed with the EAR and Sanya VHF radar in Southeast Asia, J. Geophys. Res., 118, 6544-6557, doi:10.1002/jgra.50581, 2013.

Liu, L., Chen, Y., Le, H., Kurkin, V. I., Polekh, N. M., and Lee, C.C.: The ionosphere under extremely prolonged low solar activity, J. Geophys. Res., 116, A04320, doi:10.1029/2010ja016296, 2011.

Lynn, K., Otsuka, Y., and Shiokawa, K.: Simultaneous observations at Darwin of equatorial bubbles by ionosonde-based range/time displays and airglow imaging, Geophys. Res. Lett., 38, L23101, doi:10.1029/2011GL049856, 2011.

Nicolls, M. J., Kelley, M. C., Vlasov, M. N., Sahai, Y., Chau, J. L., Hysell, D. L., Fagundes, P. R., Becker-Guedes, F., and Lima, W. L. C.: Observations and modeling of post-midnight uplifts near the magnetic equator, Ann. Geophys., 24, 1317-1331, doi:10.5194/angeo-24-1317-2006, 2006.

Ning, B., Hu, L., Li, G., Liu, L., and Wan, W.: The first time observations of low-latitude ionospheric irregularities by VHF radar in Hainan, Sci. China Ser. A, 55, 1189-1197, doi:10.1007/s11431012-4800-2, 2012.

Otsuka, Y. and Ogawa, T.: VHF radar observations of nighttime $F$ region field-aligned irregularities over Kototabang, Indonesia, Earth Planets Space, 61, 431-437, 2009.

Otsuka, Y., Shiokawa, K., Ogawa, T., and Wilkinson, P.: Geomagnetic conjugate observations of medium-scale traveling ionospheric disturbances at midlatitude using allsky airglow imagers, Geophys. Res. Lett., 31, L15803, doi:10.1029/2004g1020262, 2004.

Patra, A., Phanikumar, D., and Pant, T.: Gadanki radar observations of $\mathrm{F}$ region field-aligned irregularities during June solstice of solar minimum: First results and preliminary analysis, J. Geophys. Res., 114, A12305, doi:10.1029/2009JA014437, 2009.

Sahai, Y., Fagundes, P. R., and Bittencourt, J. A.: Transequatorial $F$ region ionospheric plasma bubbles: solar cycle effects, J. Atmos. Sol.-Terr. Phy., 62, 1377-1383, doi:10.1016/S13646826(00)00179-6, 2000.
Saito, A., Nishimura, M., Yamamoto, M., Fukao, S., Kubota, M., Shiokawa, K., Otsuka, Y., Tsugawa, T., Ogawa, T., Ishii, M., Sakanoi, T., and Miyazaki, S.: Traveling ionospheric disturbances detected in the FRONT Campaign, Geophys. Res. Lett., 28, 689-692, doi:10.1029/2000GL011884, 2001.

Shiokawa, K., Ihara, C., Otsuka, Y., and Ogawa, T.: Statistical study of nighttime medium-scale traveling ionospheric disturbances using midlatitude airglow images, J. Geophys. Res., 108, 1052, doi:10.1029/2002ja009491, 2003a.

Shiokawa, K., Otsuka, Y., Ihara, C., Ogawa, T., and Rich, F. J.: Ground and satellite observations of nighttime medium-scale traveling ionospheric disturbance at midlatitude, J. Geophys. Res., 108, 1145, doi:10.1029/2002ja009639, 2003 b.

$\mathrm{Su}$, S. Y., Chao, C. K., and Liu, C. H.: On monthly/seasonal/longitudinal variations of equatorial irregularity occurrences and their relationship with the postsunset vertical drift velocities, J. Geophys. Res., 113, A05307, doi:10.1029/2007ja012809, 2008.

Tsunoda, R. T. and Cosgrove, R. B.: Coupled electrodynamics in the nighttime midlatitude ionosphere, Geophys. Res. Lett., 28, 4171-4174, doi:10.1029/2001gl013245, 2001.

Van Dierendonck, A. J. and Hua, Q. Y. D.: Crystal oscillator noise effects on the measurement of ionospheric phase scintillation using GPS, in: Proceedings of the 1998 Ieee International Frequency Control Symposium, Proceedings of the Ieee International Frequency Control Symposium, 27-29 May 1998, IEEE, New York, USA, 298-305, 1998.

Whalen, J. A.: The linear dependence of $\mathrm{GHz}$ scintillation on electron density observed in the equatorial anomaly, Ann. Geophys., 27, 1755-1761, doi:10.5194/angeo-27-1755-2009, 2009.

Xiao, S., Xiao, Z., Shi, J., Zhang, D., Feng, X., Hao, Y., and Huang, W.: Observational facts in revealing a close relation between acoustic-gravity waves and midlatitude spread-F, J. Geophys. Res., 114, A01303, doi:10.1029/2008ja013747, 2009.

Xiao, Z., Xiao, S., Hao, Y., and Zhang, D.: Morphological features of ionospheric response to typhoon, J. Geophys. Res., 112, A04304, doi:10.1029/2006ja011671, 2007.

Yokoyama, T., Hysell, D. L., Otsuka, Y., and Yamamoto, M.: Threedimensional simulation of the coupled Perkins and Es-layer instabilities in the nighttime midlatitude ionosphere, J. Geophys. Res., 114, A03308, doi:10.1029/2008JA013789, 2009.

Yokoyama, T., Pfaff, R., Roddy, P., Yamamoto, M., and Otsuka, Y.: On postmidnight low-latitude ionospheric irregularities during solar minimum: 2. C/NOFS observations and comparisons with the Equatorial Atmosphere Radar, J. Geophys. Res., 116, A11326, doi:10.1029/2011JA016798, 2011a.

Yokoyama, T., Yamamoto, M., Otsuka, Y., Nishioka, M., Tsugawa, T., Watanabe, S., and Pfaff, R.: On postmidnight low-latitude ionospheric irregularities during solar minimum: 1. Equatorial Atmosphere Radar and GPS-TEC observations in Indonesia, J. Geophys. Res., 116, A11325, doi:10.1029/2011JA016797, 2011b. 